Research Paper

\title{
Efficacy of Shikonin against Esophageal Cancer Cells and its possible mechanisms in vitro and in vivo
}

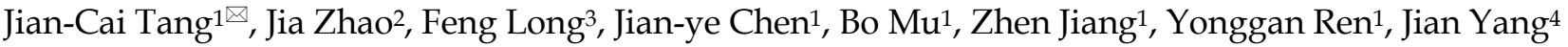 \\ 1. Department of Biochemistry; \\ 2. School of Pharmacy; \\ 3. Department of Pharmacy, Nan Chong Central Hospital; \\ 4. Pathogenic Biology and Immunology Experiment Teaching Center, North of Si Chuan Medical University, China. \\ *Jian-Cai Tang and Jia Zhao contributed equally to this work.
}

$\square$ Corresponding author: Jian-Cai Tang, PhD; North of Si Chuan Medical College, Fu jiang Road 234, Shun Qing District, Nan Chong, Sichuan province, China Post code: 637000; E-mail: tangjiancai@nsmc.edu.cn

(c) Ivyspring International Publisher. This is an open access article distributed under the terms of the Creative Commons Attribution (CC BY-NC) license (https://creativecommons.org/licenses/by-nc/4.0/). See http://ivyspring.com/terms for full terms and conditions.

Received: 2017.05.28; Accepted: 2017.08.22; Published: 2018.01.01

\begin{abstract}
Increasing evidences indicate that shikonin can suppress the tumor growth. However, the mechanisms remain elusive. In the present study, we investigated the effects and mechanisms of shikonin against esophageal cancer. The expression of hypoxia inducible factor $1 \alpha(\mathrm{HIFl \alpha})$ and pyruvate kinase M2 (PKM2) in esophageal cancer tissues and cells was detected by immunohistochemistry and Western blot. CCK-8 was used to examine the esophageal cancer cell viability. Apoptosis and cell cycle were analyzed by flow cytometry. The expression of EGFR, PI3K, Akt, p-AKT, mTOR, HIFla and PKM2 was detected by Western blot. EC109/pkm2 was established by lentivirus transducer. Ec109 tumor model was founded to observe the antitumor effect of shikonin in vivo.

We found that HIFl $\alpha$ and PKM2 protein expression levels were higher in esophageal cancer tissues and cells than normal esophageal tissues and cells. Shikonin reduced esophageal cancer cells viability and induced cell cycle arrest and apoptosis. Shikonin decreased EGFR, PI3K, P-AKT, HIFl $\alpha$ and PKM2 expression. Overexpression of PKM2 could enhance resistance of esophageal cancer cells to shikonin. In vivo we found that shikonin reduced tumor burden, inducing cell arrest and apoptosis. Taken together, shikonin has a significant antitumor effect in the esophageal cancer by regulating HIFla/PKM2 signal pathway.
\end{abstract}

Key words: Shikonin, Esohpageal cancer, hypoxia inducible factor 1a, Pyruvate kinase M2, CyclinD1

\section{Introduction}

Esophageal cancer is the sixth leading cause of cancer-related worldwide, including two main types: esophageal squamous cell carcinoma (ESCC) and Esophageal adenocarcinoma (EAC) [1, 2]. Most subtype of EC is ESCC which accounts for $87 \%$ of cases in 2012[3]. Although the development of surgical techniques, radiotherapy and chemotherapy, the survival rate of five years is still about $19 \%$ [4]. Thus, it is urgent to find new drugs to treat esophageal cancer.

Tumor metabolism is an emerging hallmark of cancer cells. The reprogramming of cellular metabolism is critical to maintain survival and limitless proliferation of cancer cells under severe conditions during development. Acrobic glycolysis [5] is a vital biological characteristics of cancer, not only because it provides tumor cells with energy and raw materials for the synthesis of biomacromolecular but also have close relationship with anti-apoptosis, invasion, metastasis and drug resistance of tumor cells. Hypoxia-inducible factor-1 (HIF-1), a molecular determinant of responses to hypoxia in mammalian cells, is a heterodimer consisting of two subunits, an oxygen-sensitive HIF-1a and a constitutively expressed HIF-1 $\beta$. HIF-1 activity in tumors depends on availability of the HIF-1a subunit, the levels of 
which increase under hypoxic conditions and through activation of oncogenes and/or inactivation of tumor suppressor genes. HIF1a $[6,7]$ is required for hypoxia-induced cell cycle arrest, the mechanism that the enhancing stability of HIF-1a protein regulated cyclinD1 expression under hypoxia condition. HIF-1a [8] induced G0/G1 phase arrest may account for the resistance of cancer cells to some certain chemotherapeutic agents that target DNA synthesis in $S$ phase. Elevated expression of HIF-1a is usually detected in various solid tumors including colon [9], breast [10], prostate [11], and lung cancers [12], and is associated with resistance [13] to therapies and poor prognosis in multiple cancers. PKM2 [14-16], a downstream molecular of HIF1a, is key enzyme invoved in the aerobic glycolysis of cancer cells. Inhibiting PKM2 can result in the decreased intacellular ATP and increased intake of anticarcinogen, and further promotes therapeutic sensentivity.

Shikonin [17] is a natural naphthoquinone isolated from a traditional Chinese medicine, the plant Lithospermum erythrorhizon, (zicao). Many studies [18-20] have proved that shikonin can suppress multiple types cancers, including breast cancer, hepatocellular cancer etc. However, the effects and mechanisms of shikonin against esophageal cancer remain elusive.

In the present study, our aim is to investigate the effects and mechanisms of shikonin against esophageal cancer. PKM2 and HIF1 were examined in esophageal cancer and normal esophageal tissue by immunochemistry. CCK-8 was used to detect the effect of shikonin on the esophageal cancer cells. EGFR, PI3K, AKT, P-AKT, mTOR, PKM2 and HIF1 $\alpha$ were evaluated by western blot after shikonin treatment. Ec109 tumor model was established to observe the antitumor effect of shikonin in vivo. Our study identified that shikonin suppressed the proliferation of esophageal cancer cells by regulating HIF-1a/PKM2 signal pathway in vitro and in vivo.

\section{Material and Methods}

\section{Reagents}

Shikonin was purchased from Sigma (S7576). Antibodies against EGFR, PI3K, AKT, P-AKT and mTOR were purchased from Cell signaling Technology (USA). Antibodies against HIF-1a and PKM2 were purchased from ABCAM(USA). $\beta$-actin and Rabbit secondary antibody were purchased from ZSGB-BIO(China). CCK-8 was purchased from Dojindo (Japan). Cell cycle kit and cell apoptosis kit were purchased from Boster (China).

\section{Samples and immunohistochemistry}

Between October 2014 and October 2015, a total of 20 normal esophageal tissues, 40 early esophageal cancer and 40 advanced Esophageal cancer tissues were obtained from patients admitted at Affiliated Hospital of North of Sichuan Medical college (Si Chuan, China). All esophageal cancer cases were confirmed as ESCC through pathological diagnosis.

Tumor tissues were embedded and sectioned $(4-5 \mu \mathrm{m})$. Then, sections were dewaxed in xylene, rehydrated through decreasing concentrations of ethanol, and washed in PBS. Next, Immunostaining was undertaken using anti-human HIF1a (1:100) and PKM2 (1:100) monoclonal antibody at $4^{\circ} \mathrm{C}$. After incubation with the appropriate HRP-labeled second antibody, immunostaining was performed by $\mathrm{DAB}$ according to manufacture instruction. Immunostaining results were evaluated to be graded $0,1,2$ and 3 according to the extent of staining by two pathologists.

\section{Cell cultures and chemicals}

Human Esophageal cancer cell EC109, EC9706 cells and Human Esophageal Epithelial Cells (HECC) were provided from ATCC. Cells were grown in 1640 supplemented with $10 \%$ (vol/vol) fetal bovine serum and were maintained in a humidified chamber at $37^{\circ} \mathrm{C}$.

\section{CCK-8 assay}

Cell proliferation was determined by CCK- 8 assay. Cells were seeded at $5 \times 10^{3}$ cells/well in 96-well plates and cultured for overnight at $37^{\circ} \mathrm{C}$. Cells were treated with shikonin of different concentrations for $24 \mathrm{~h}$ and $48 \mathrm{~h}$. Absorbance was determined at $450 \mathrm{~nm}$ using an enzyme-linked immunosorbent assay reader.

\section{Western blot}

The total protein was exacted from cells according to standard procedures, then separated by SDS-PAGE gel electrophoresis and transferred to PVDF membranes. The membranes were blocked, then incubated with primary antibodies overnight, followed by incubation with appropriate HRP-conjugated secondary antibodies (1:5000 dilutions). The blots were detected using ECL chemiluminescence according to the manufacturer's instructions.

\section{RT-PCR}

Total RNA was isolated from cells by using Trizol reagent. RT-PCR was performed using SYBR premix EX Tag reagents by two steps according to manufacturer's instructions. The specific primers were as follows: 
ß-actin: forward: 5'-ATGCCAACACAGTGTTGTCTG G-3'; reverse: 5'-TACTCCTGCTTGCTGATCCACAT$3^{\prime}$.

PKM2 forward: 5'-TGTCTGGAGAAACAGCCA AG-3'; Reverse: 5'-TCCTCGAATAGCTGCAAGTG$3^{\prime}$.

\section{Flow cytometry}

Ec109 and EC9706 Cells were seeded in six-well plates at $4 \times 10^{5}$ per well. Cells were treated with varying concentration of shikonin for $24 \mathrm{~h}$. Cell cycle was assayed by measuring DNA fragment staining with PI. Cell apoptosis was evaluated by Annexin-v-FITC and PI staining. All procedures were conducted according to the instructions of cell cycle kit and cell apoptosis kit.

\section{Establishment of stably transduced cell lines}

To observe the role of PKM2 for shikonin against esophageal cancer cells, limited dilution was used to establish monoclonal cell lines. The plasmid pLV-201-CMV-EGFP/PKM2 constructed and Lentivirus packaging (p-LV201-200, pLV201-050) were performed by GeneCopoeia company (Guang Zhou, China).

To transduce the cells, the total volume of $300 \mu \mathrm{L}$ medium was added which contained $100 \mu \mathrm{L}$ of the packaged viral supermatant (MOI=1 0). After $24 \mathrm{~h} 500$ $\mu l$ medium without virus solution were replaced. The fluorescence emission of cells was observed $72 \mathrm{~h}$ after transduction. $100 \mu$ Lof cell suspension containing single cells were added to each well in 96-well plates. Cells were conventional cultured for 20 days to select good positive clones with good growth state, and then picked for expanding culture. After expanding culture and preservation, it was named Ec109/PKM2.

\section{Animal model}

Ec109 tumor model was established to observe the antitumor effect of shikonin in vivo. Nude mice (6-six-week) were purchased from vital River Laboratory Animal Technology (Co. Ltd., Bei Jing). Mice were injected with Ec109 $\left(5 \times 10^{6}\right)$ in the dorsal area. The mice were divided randomly into two groups: shikonin treatment group $(20 \mu \mathrm{g} /$ dose/day) and control group (injected with DMSO) after 7days (each group has 7 mice). Treatment was performed for 21 days. Tumor volumes were evaluated every 3 days according to the following formula:

$$
\mathrm{v}=0.52 \times \text { width }^{2} \times \text { length }
$$

\section{Evaluation of apoptosis}

Tumor cells apoptosis was assessed using
TUNEL (DeadEnd ${ }^{\mathrm{TM}}$ Fluorometric TUNEL System, Promega, Madison, USA) according to the manufacturer's instructions.

\section{Statistical analysis}

All data were analyzed with SPSS 17.0 software system. Significance of the differences was considered at $\mathrm{P}<0.05$.

\section{Results}

\section{High expression of PKM2 and HIF $1 \alpha$ in Esophageal cancer tissues and cells}

The expression of PKM2 and HIF1a was detected by immunochemistry in normal esophageal tissues, early esophageal cancer tissues and advanced esophageal cancer tissues. The results showed that the expression of PKM2 and HIF1a increased significantly in early and advanced esophageal cancer tissues compared with normal esophageal tissues. (Fig.1A,B) Moreover, the expression of PKM2 and HIF1a was examined by Western blot in Human Esophageal Epithelial Cells(HECC), EC109 and EC9706. Our results displayed that the expression of PKM2 and HIF1a rose significantly in the EC109 and EC9706 compared with Human Esophageal Epithelial Cells (HECC). (Fig.1 C) These data indicate that there are high expressions of PKM2 and HIF1a in Esophageal cancer tissues and cells.

\section{Shikonin inhibits esophageal cancer cells proliferation}

In order to examine the effect of shikonin on the proliferation of esophageal cancer cells, CCK-8 was used to detect the viability of esophageal cancer cells. The results displayed that shikonin decreased the viability of EC109 and EC9706 in a dose and time-dependent. (Fig.2A,B)

\section{Shikonin induces cell cycle arrest and apoptosis in esophageal cancer cells}

To explain the mechanism of shikonin inhibiting the proliferation of esophageal cancer cells, we detect the cell cycle and apoptosis after shikonin treatment. We found that shikonin induced cell cycle arrest in EC109 and EC9706. (Fig.3 A) and increased the proportion of apoptosis. (Fig.3B)

The expression level of Capase3, Capase9 and cyclinD1 was examined after shikonin treatment by Western Blot. The results showed that shikonin activated caspase3, and decreased the expression of capase 9 and cyclinD1. (Fig.3C) 
A

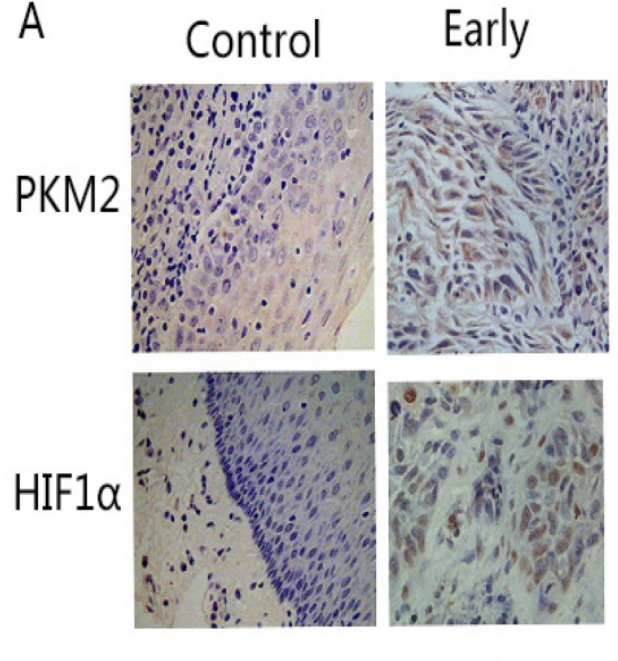

C

\section{Advanced}
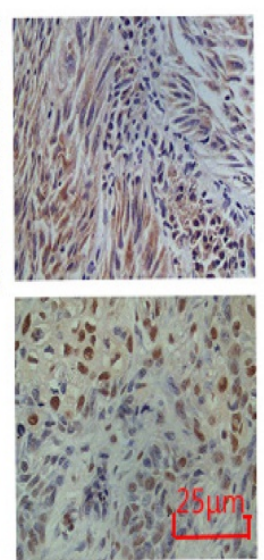

B
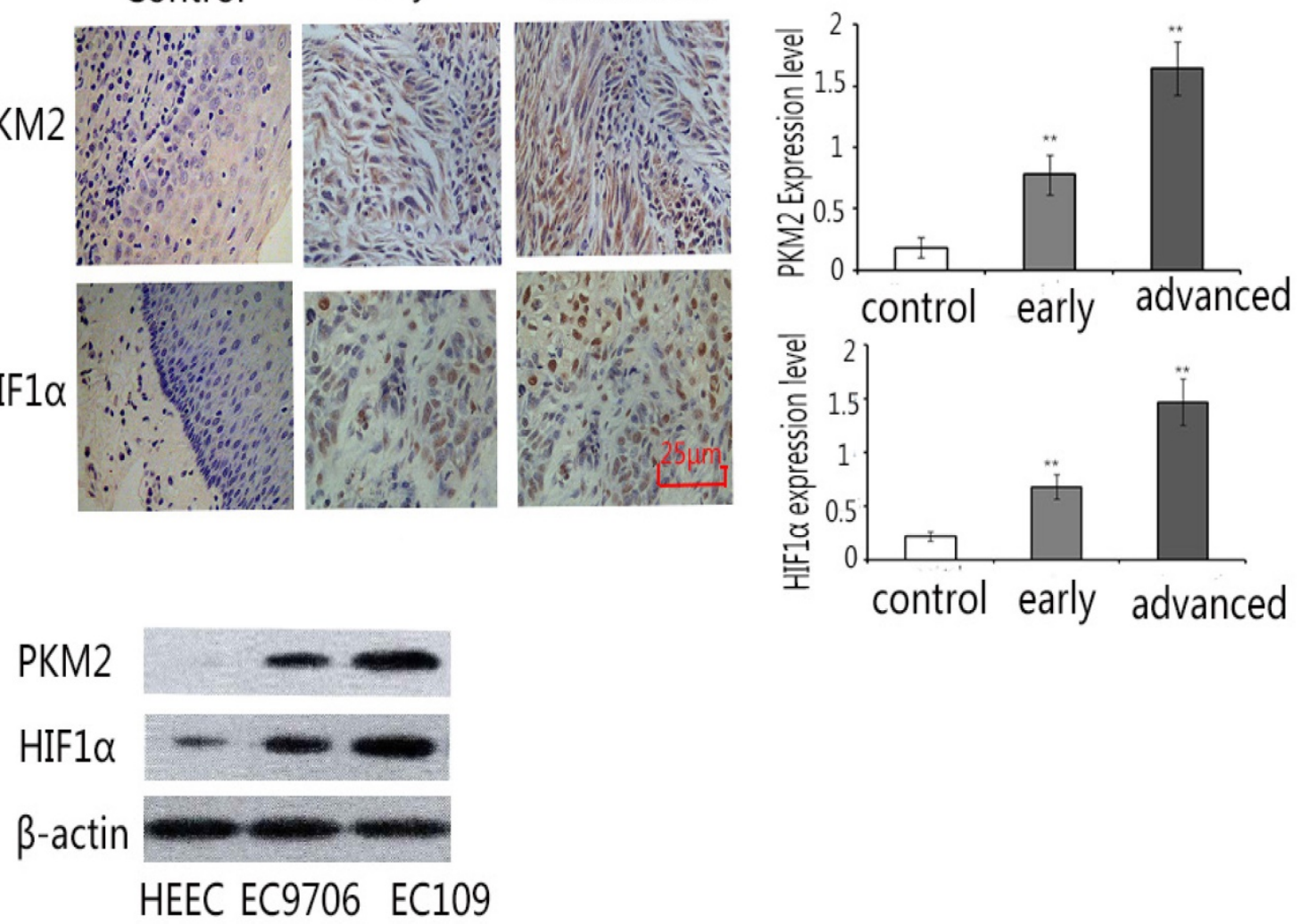

Fig. 1. The expression of Pkm2 and HIF1a in normal esophageal, early esophageal cancer, advanced esophageal cancer tissues and esophageal cancer cells. Samples were detected by immunohistochemical staining according to standard protocol. A. Representative images of PKM2 staining (left), the average expression level of PKM2 (right). B. Representative images of HIFla staining (left), the average expression level of HIFla (right). The results exhibited that the expression of PKM2 and HIFla increased in early and advanced esophageal cancer tissues than normal esophageal tissues. ( $\times 200)$. C. the expression of PKM2 and HIFla in Human Esophageal Epithelial Cells, EC109 and EC9706 by Western blot. The results showed that the expression of PKM2 and HIF1 a rose significantly in the EC109 and EC9706 compared with Human Esophageal Epithelial Cells (HECC). **P<0.01

A

Eca109

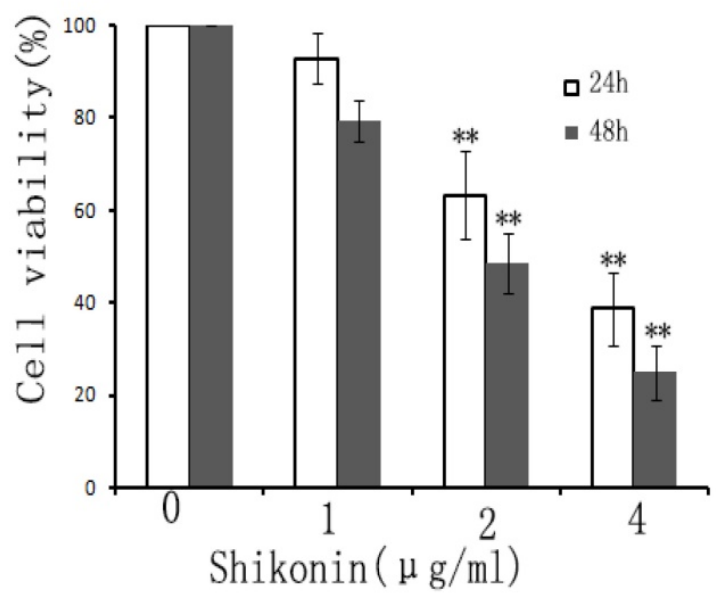

B

\section{Ec9706}

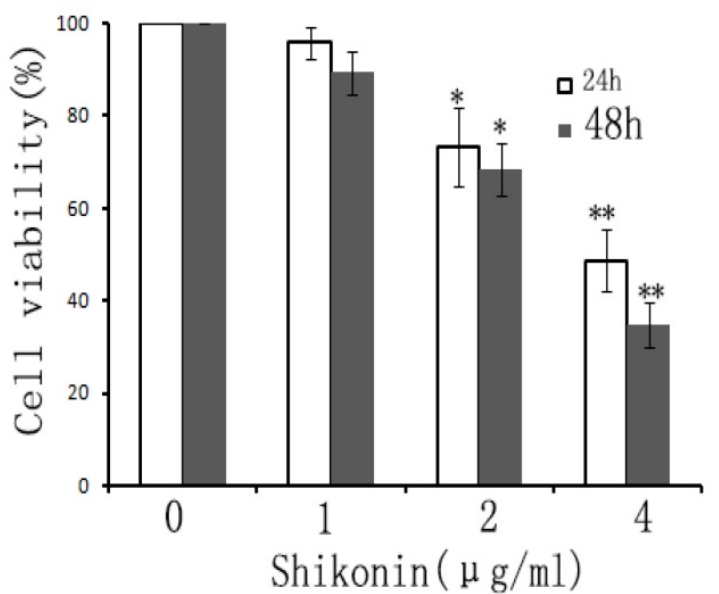

Fig. 2. Shikonin inhibited the proliferation of esophageal cancer cells. Esophageal cancer cells were treated with different concentrations of shikonin $(0,1,2,4 \mu \mathrm{g} / \mathrm{ml})$ for $24,48 \mathrm{~h}$. CCK-8 was used to detect the cell viability. The results showed that Shikonin decreased EC109 (A) and EC 9706 (B) cell viability in a time and dose-dependent manner. Data was described as the means \pm SD of three independent experiments. $(* P<0.05, * * P<0.01)$ 

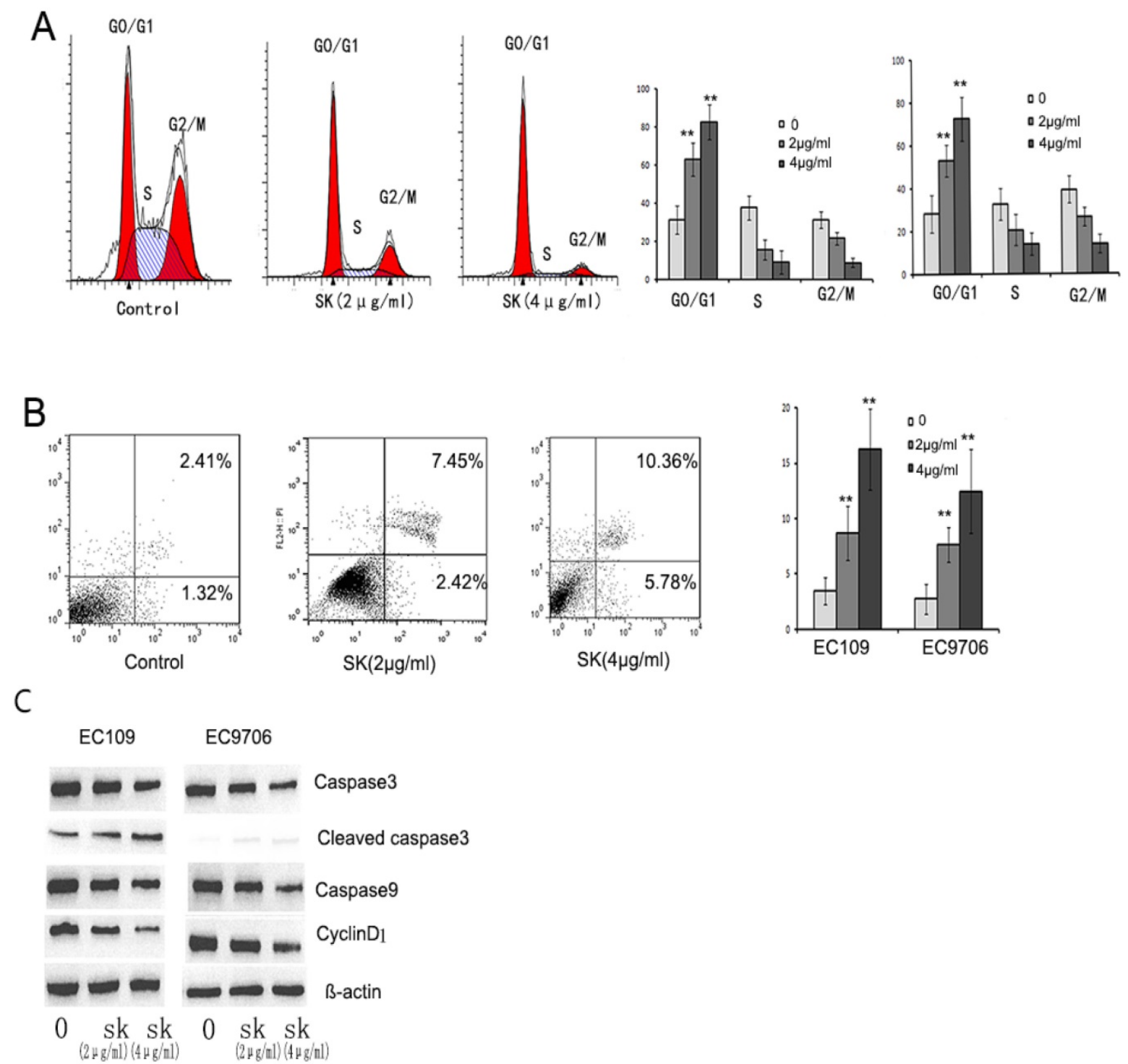

Fig. 3. Shikonin induced cell cycle arrest and apoptosis. EC109 and EC 9706 cells were treated with different concentration of shikonin $(2,4 \mu g / \mathrm{ml})$ for $24 \mathrm{~h}$. Cell cycle and apoptosis were analyzed by Flow cytometry. A. Representative images of cell cycle(left), the proportions of different phases of cell cycle(Right). B. Representative images of apoptosis (Left). The proportion of apoptosis (Right). C. The apoptosis and cell cycle related-proteins were detected by Western blot. The results showed that shikonin induced cell cycle arrest G0/Gl phase and increased the proportion of apoptosis in esophageal cancer cells. Furthermore, shikonin activated caspase 3 and decreased the expression of caspase 9 and cyclinD1. ${ }^{*} \mathrm{p}<0.05,{ }^{*} \mathrm{p}<0.01$

\section{Shikonin decreases the EGFR, PI3K, P-AKT, mTOR, HIF I a, PKM2 expression}

To explore the possible molecular mechanisms for antitumor effect of shikonin, we examined the expression of EGFR, PI3K, P-AKT, mTOR, HIF1a, PKM2 in esophageal cancer cells after shikonin treatment. The results demonstrated that shikonin reduced evidently the expression of HIF1a, PKM2, PI3K, P-AKT, mTOR. in EC109 and EC9706.(Fig.4A,B)

\section{Overexpression PKM2 increases resistance of esophageal cancer cells to shikonin}

To investigate whether PKM2 may be the key factor for shikonin against esophageal cancer proliferation, Ec109/PKM2 was established according to previous methods. RT-PCR and Western blot were used to identify the high expression of PKM2 in Ec109/PKM2. We found that the expression of PKM2 increased evidently in Ec109/PKM2 compared to Ec109. (Fig.5 A,B)

Next, we examined the effect of shikonin on Ec109/PKM2. The results showed that overexpression PKM2 increased shikonin resistance in esophageal cancer cells. (Fig.5C) Furthermore, the expression of cyclinD1 decreased evidently after shikonin in Ec109/PKM2. (Fig.5D, E) These results indicate that PKM2 may play an important role by regulating the expression of CyclinD1 in the proliferation of esophageal cancer cells. 
A
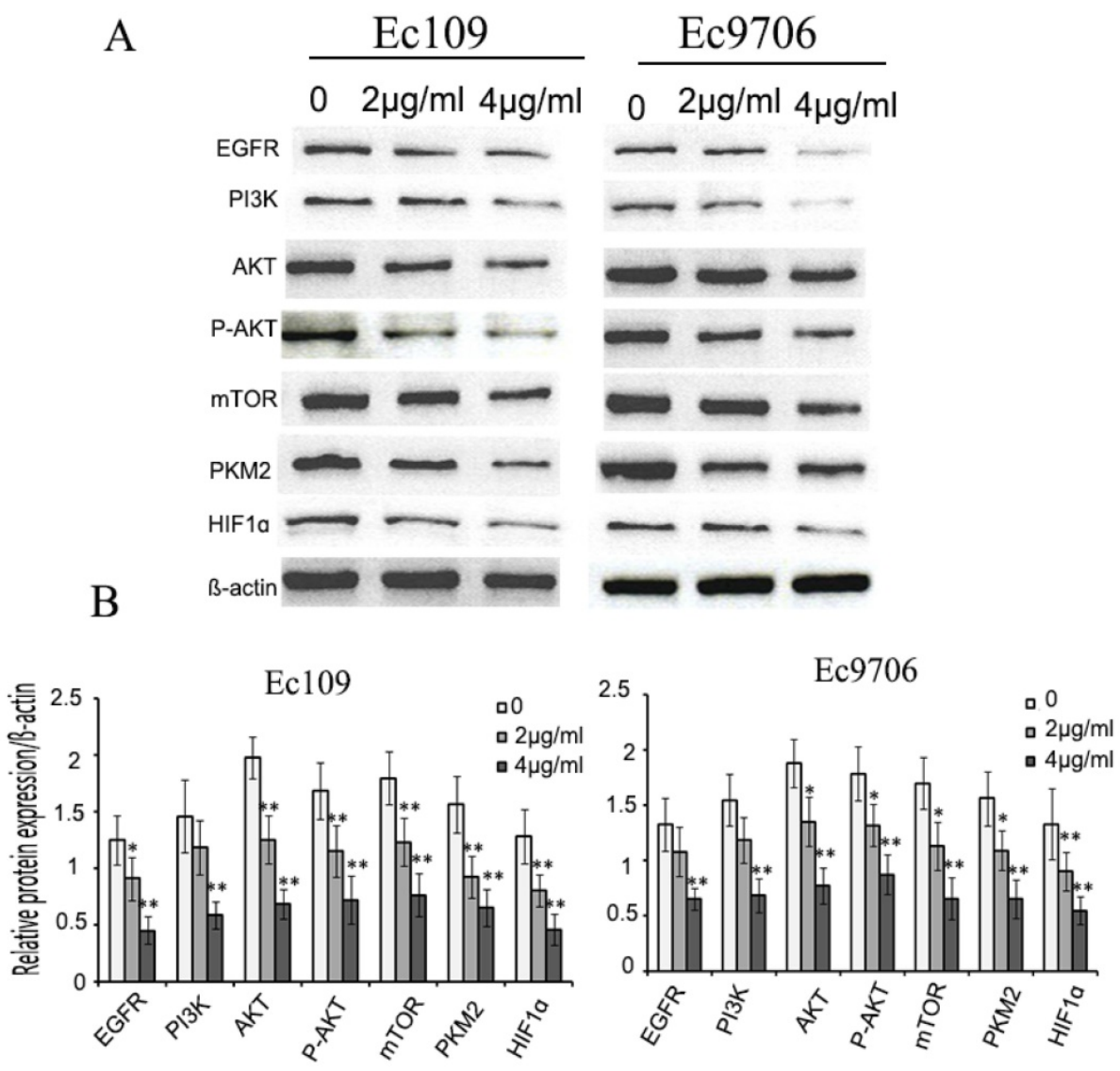

Fig. 4. Shikonin inhibited EGFR/PI3K/AKT signal pathway. Ec109 and EC9706 cells were treated with shikonin(0,2,4 $\mu \mathrm{g} / \mathrm{ml})$ for $24 \mathrm{~h}$ and the total protein were extracted, then the expression of EGFR, PI3K, AKT, P-AKT, mTOR, PKM2 and HIFIa were examined by Western blot. The results showed that shikonin decreased the expression of EGFR, PI3K, AKT, P-AKT, mTOR, PKM2 and HIFIa.
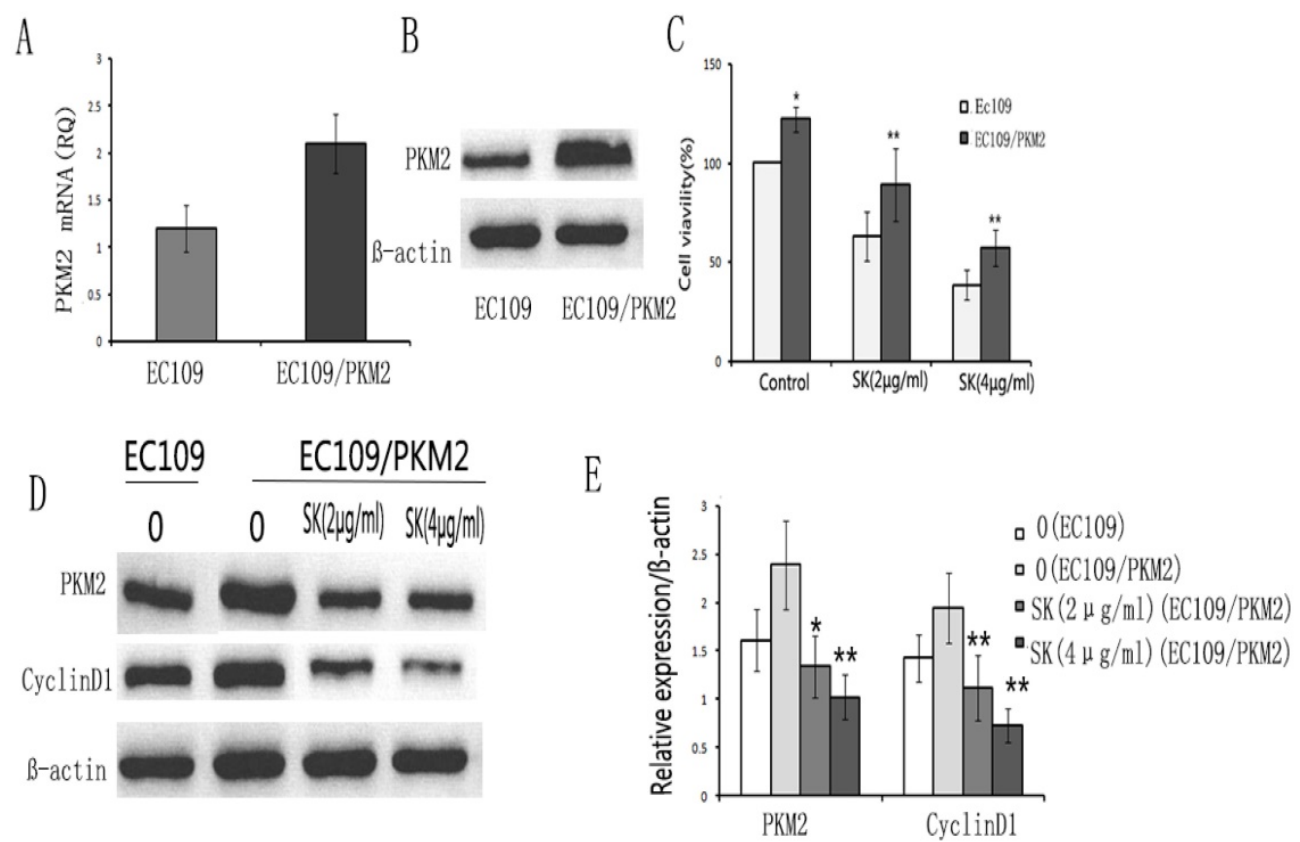

Fig. 5. PKM2 overexpression increased resistance of EC109 to shikonin. EC109/PKM2 stable cell was established by transducing Lentivirus plasmid according to previous methods. RT-PCR(A) and Western blot(B) showed that the expression of PKM2 was higher in EC109/PKM2 cell than EC109 cell. C. Cell viability was detected by CCK-8 assay. The results showed cell viability and resistance of EC109/PKM2 cells to shikonin increased evidently compared to EC109 cells. D. Shikonin decreased the expression of PKM2 and cyclinD1 in EC109 and Ec109/pkm2 cells. ${ }_{p}<<0.05,{ }^{*}{ }^{*}<0.01$ 


\section{Antitumor effect of shikonin in vivo}

To further evaluate the antitumor effect of shikonin in vivo, Ec109 tumor model was established in nude mice. The results exhibited that shikonin decreased the tumor burden after shikonin administration.(Fig.6A,B) Furthermore, we also found that shikonin reduced the expression of PKM2, HIF1a and cyclinD1 in tumor tissues.(Fig.6C,D) Moreover, there were more apoptotic cells by TUNEL staining after shikonin treatment. (Fig.6E,F) Taken together, these results showed that shikonin has a powerful antitumor effect in vivo.

\section{Discussion}

In the present study, our results showed the expression of PKM2 and HIF1a increased in early and advanced esophageal cancer tissues, and found that shikonin inhibited the proliferation of esophageal cancer cells in vitro and in vivo. The antitumor mechanisms of shikonin may be associated with regulating HIF1a/PKM2 signal pathway.
A

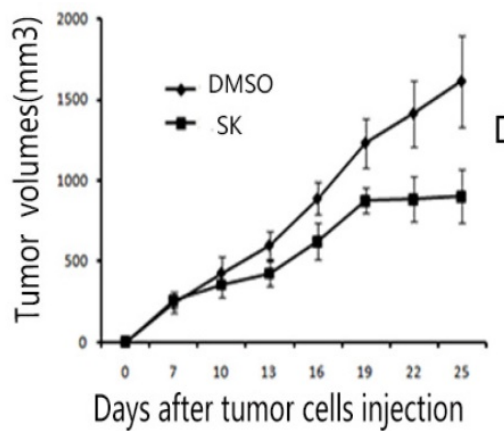

B

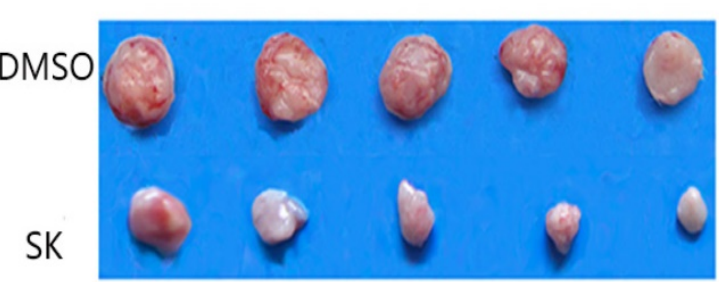

D
C

PKM2

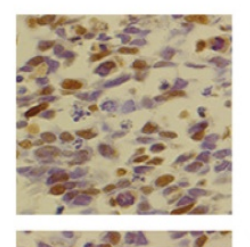

HIF1 $\alpha$

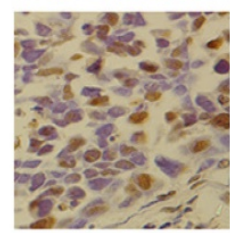

CyclinD1

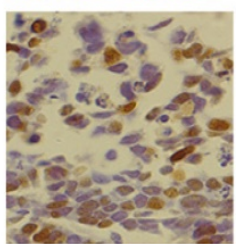

$E$
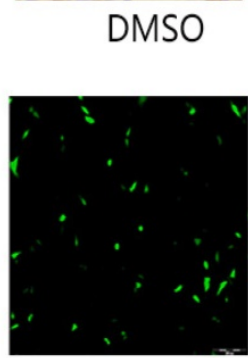

DMSO
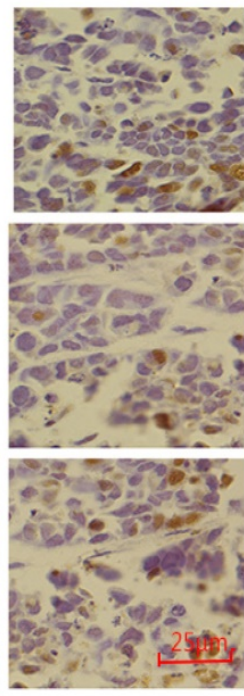

SK

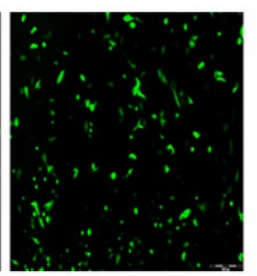

SK

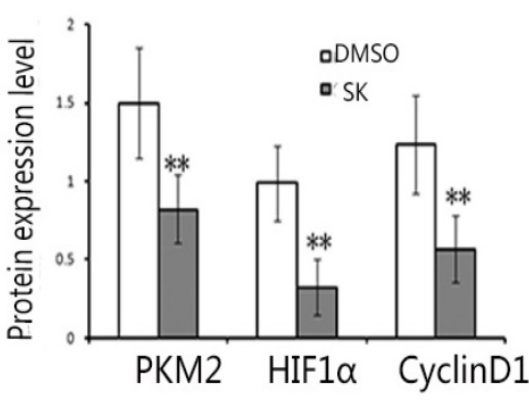

F

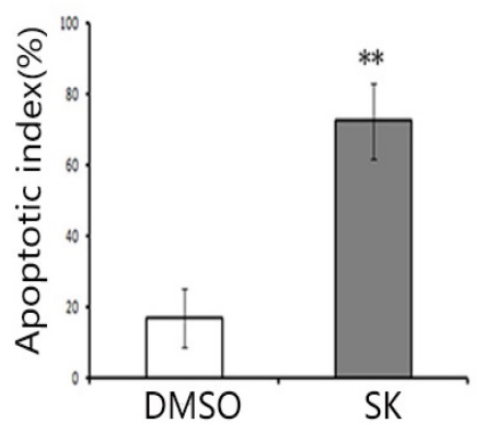

Fig. 6. Shikonin suppressed the proliferation of EC109 cells in vivo. EC109 tumor model was established to observe the antitumor effect of shikonin in vivo. Mice were treated with DMSO or shikonin $(300 \mathrm{mg} / \mathrm{Kg} /$ day $)$ for 21 days at the same time $(\mathrm{n}=7$ per group). Two mice in experimental group were dead at 13 and 18 days after tumor cells injection. A. The tumor growth curve. The results showed shikonin suppressed the tumor growth ( $\mathrm{n}=7$, $\mathrm{p}<0.01$ ). Data are shown as mean \pm SD. B. Representation of photos of tumors from mice bearing Eca 109 receiving different treatments. C. Typical graph of IHC staining of PKM2, HIF1 a and cyclinD1 in tumor tissues. $(\times 200)$. D. The average staining intensities of PKM2, HIFla and cyclinD1. Staining intensities were graded as $0,1,2,3$ by two pathologists, respectively. The results showed that Shikonin treatment significantly decreased the expression of PKM2, HIF1 a and cyclinD1. s. (**P<0.01) (E) Typical sections of apoptosis from tumor tissue. F. apoptotic index within tumor tissues. Shikonin showed a significant increase of apoptotic cells in tumor tissues compared to the control $(* * P<0.01)$. Data are expressed as the mean apoptotic index \pm SD. 
Many studies [21-23] have found shikonin had antitumor effect in various cancer types, the mechanisms of which were given on the aspects of induction of apoptosis, necrosis, acting on proteasome, protein tyrosine kinase and anti-angiogenesis. The molecular mechanisms mainly included stimulating ROS generation [22] in the mitochondria, inhibiting the activities of DNA topoisomerases, increasing expression of p53[23, 24] and inhibition of cancer cell glycolysis via targeting PKM2, polo-like kinase1 (PLK1) and protein tyrosine kinase (PTK) [19, 25]. Compared with previous studies, our study found that shikonin inhibited HIF1a/pkm2/CyclinD1 signal pathway to regulate the proliferation of esophageal cancer cells. To our knowledge, it is the first time to prove that HIF1a/ PKM2/cyclinD1 signal pathway may be a new target of shikonin against esophageal cancer cells.

HIF-1a [12, 26, 27] has been an important role in the regulation of tumor proliferation, which is shown to activate multiple target genes that involve in crucial aspects of cancer biology, including angiogenesis, glucose metabolism, cell proliferation/survival and apoptosis. Increased the expression of HIF1a is connected with rising risk of mortality in various types of cancers, including esophageal cancer. PI3K/AKT/HIF1a signal pathway plays an important role in the regulation of tumor glucose metabolism. PKM2, a key downstream molecular of HIF-1a is key enzyme involved in the aerobic glycolysis of cancer cells. Previous studies [28, 29] have proved that inhibiting PKM2 can decrease intracellular ATP, and then suppress the proliferation of cancer cells. PKM2 may be a new biomarker for many cancers, such as lung cancer, breast cancer. In the present study, we found esophageal cancer tissue was high expression of HIF1a and PKM2 compared to normal esophageal tissue. Furthermore, our results showed that shikonin inhibited the esophageal cancer cells proliferation, accompanying with decreased the HIF1a and PKM2 expression indicating that shikonin suppressed the cancer cells proliferation via HIF1a/PKM2 signal pathway.

HIF1a is a downstream molecular of EGFR/PI3K/AKT/mTOR signal pathway. Previous studies [21,30] have proved that shikonin can inhibit EGFR-signaling to suppress cell proliferation in various cancers cells. PI3K is an important downstream molecular of EGFR. In the current study, our results showed that shikonin suppressed the EGFR/PI3K/AKT/mTOR signal pathway, decreasing the expression of HIF1a and PKM2.

CyclinD1 [31, 32], a target gene of HIF1a, is key mediator of cell growth and proliferation in cancer cells. Some researches $[33,34]$ had proved that HIF1a regulated the expression of cyclin D1, and then promoted G1/S cell cycle transition and cell proliferation, which the mechanisms may be associated with transcriptional coactivator for Pkm2. Specifically, Pkm2[35] interacting with HIF1a promotes the transactivation of HIF1a target genes. In the current study, we found that shikonin decreased the expression of cylcinD1, indicating that shikonin reduced the esophageal cancer cells proliferation by suppression HIF1a / PKM2/cyclin D1 pathway.

To prove that the role of PKM2 in mediating antitumor effect of shikonin, EC109/PKM2 cell was established by Lentivirus transduced. The results showed that the resistance of EC109/pkm2 to shikonin was obviously enhanced compared with EC109 cells. These results suggest that shikonin inhibits esophageal cancer cells by inhibiting HIF1a / PKM2. Furthermore, we found that shikonin reduced tumor growth in vivo, displaying that shikonin may be a new drug against ESCC. Moreover, Shikonin increased tumor cells apoptosis, decreasing PKM2, HIF1a and cyclinD1 expression in vivo. These are consistent with previous in vitro results.

Taken together, our research proves that shikonin inhibits the proliferation of esophageal cancer cells in vitro and in vivo. Antitumor effect of shikonin may be connected with inducing apoptosis and cell cycle arrest by inhibiting the HIF1a/PKM signal pathway. These findings indicate that shikonin may be a new drug for treating esophageal cancer.

However, our results have some limitations. Specifically, shikonin has serious side effects in vivo. We found that mice have weight loss, spirits drooping, even dead after shikonin administration. How to reduce adverse effects needs to further study. Therefore, more experiments are necessary to transform to a clinical trials.

\section{Acknowledgments}

This work is supported by the Research Fund for the Doctoral Program of Higher Education of North of Sichuan Medical college (CBY13-QD-04), Major Project of Education Department in Sichuan (10ZA168) and Applied Basic Research Programs of Science and Technology Commission Foundation of Si Chuan Province (2013JY0071).

\section{Competing Interests}

The authors have declared that no competing interest exists.

\section{References}

1. Munoz-Pinedo C, El Miiyad N, Ricci JE. Cancer metabolism: current perspectives and future directions. Cell death \& disease. 2012; 3: e248.

2. Banerjee J, Bruckbauer A, Zemel MB. Activation of the AMPK/Sirt1 pathway by a leucine-metformin combination increases insulin sensitivity in skeletal 
muscle, and stimulates glucose and lipid metabolism and increases life span in Caenorhabditis elegans. Metabolism: clinical and experimental. 2016.

3. Feng $\mathrm{Y}, \mathrm{Ke} \mathrm{C}$, Tang Q, Dong $\mathrm{H}$, Zheng $\mathrm{X}$, Lin W, et al. Metformin promotes autophagy and apoptosis in esophageal squamous cell carcinoma by downregulating Stat3 signaling. Cell death \& disease. 2014; 5: e1088.

4. Siegel RL, Miller KD, Jemal A. Cancer statistics, 2016. CA Cancer J Clin. 2016;66(1): 7-30.

5. Cairns RA, Harris IS, Mak TW. Regulation of cancer cell metabolism. Nature Reviews Cancer. 2011; 11: 85-95.

6. Park JH, Yoon J, Park B. Pomolic acid suppresses HIF1alpha/VEGF-mediated angiogenesis by targeting p38-MAPK and mTOR signaling cascades. Phytomedicine. 2016; 23(14): 1716-26.

7. Cheng T, Jian Z, Li K, Raulefs S, Regel I, Shen S, et al. In vivo functional dissection of a context-dependent role for Hif1alpha in pancreatic tumorigenesis. Oncogenesis. 2016; 5(12): e278.

8. Sanagawa A, Iwaki S, Asai M, Sakakibara D, Norimoto H, Sobel BE, et al. Sphingosine 1phosphate induced by hypoxia increases the expression of PAI1 in HepG2 cells via HIF1alpha. Mol Med Rep. 2016; 14(2): 1841-8.

9. Mladenova DN, Dahlstrom JE, Tran PN, Benthani F, Bean EG, Ng I, et al. HIF1alpha deficiency reduces inflammation in a mouse model of proximal colon cancer. Dis Model Mech. 2015; 8(9): 1093-103.

10. Jin MS, Lee H, Park IA, Chung YR, Im SA, Lee KH, et al. Overexpression of HIF1alpha and CAXI predicts poor outcome in early-stage triple negative breast cancer. Virchows Arch. 2016; 469(2): 183-90.

11. Ranasinghe WK, Baldwin GS, Bolton D, Shulkes A, Ischia J, Patel O. HIF1alpha expression under normoxia in prostate cancer--which pathways to target? J Urol. 2015; 193(3): 763-70.

12. Liao HY, Wang GP, Huang SH, Li Y, Cai SW, Zhang J, et al. HIF-1alpha silencing suppresses growth of lung adenocarcinoma A549 cells through induction of apoptosis. Mol Med Rep. 2014; 9(3): 911-5.

13. Schoning JP, Monteiro M, Gu W. Drug resistance and cancer stem cells: the shared but distinct roles of hypoxia-inducible factors HIF1alpha and HIF2alpha. Clin Exp Pharmacol Physiol 2017; 44(2): 153-61.

14. Harris I, McCracken S, Mak TW. PKM2: A gatekeeper between growth and survival. Cell research. 2011; 22: 447-9.

15. Shirai T, Nazarewicz RR, Wallis BB, Yanes RE, Watanabe R, Hilhorst M, et al. The glycolytic enzyme PKM2 bridges metabolic and inflammatory dysfunction in coronary artery disease. J Exp Med. 2017; 213(3): 337-54

16. He CL, Bian YY, Xue Y, Liu ZX, Zhou KQ, Yao CF, et al. Pyruvate Kinase M2 Activates mTORC1 by Phosphorylating AKT1S1. 2017.

17. Andujar I, Rios JL, Giner RM, Recio MC. Pharmacological properties of shikonin - a review of literature since 2002. Planta Med.2013; 79(18): 1685-97.

18. Zhu MY, Wang Rb, Zhou W, Li SS. Antitumor effect research progress of shikonin and its derivatives. Acta Pharmaceutica Sinica.2012;47(5):588-593

19. Wu H, Xie J, Pan Q, Wang B, Hu D, Hu X. Anticancer agent shikonin is an incompetent inducer of cancer drug resistance. PLoS One. 2013; 8: e52706.

20. Li W, Zhang C, Ren A, Li T, Jin R, Li G, et al. Shikonin Suppresses Skin Carcinogenesis via Inhibiting Cell Proliferation. PLoS One. 2015.

21. Li W, Liu J, Jackson K, Shi R, Zhao Y. Sensitizing the therapeutic efficacy of taxol with shikonin in human breast cancer cells. PLoS One. 2014; 9 (4) :e94079

22. Liang W, Cai A, Chen G, Xi H, Wu X, Cui J, et al. Shikonin induces mitochondria-mediated apoptosis and enhances chemotherapeutic sensitivity of gastric cancer through reactive oxygen species. Scientific Reports; $2016 ; 6: 38267$

23. Ko H, Kim SJ, Shim $\mathrm{SH}$, Chang $\mathrm{H}, \mathrm{Ha} \mathrm{CH}$. Shikonin Induces Apoptotic Cell Death via Regulation of p53 and Nrf2 in AGS Human Stomach Carcinoma Cells. Biomolecules \& Therapeutics, 2016, 24 (5) :501-509

24. Hou $Y, X u$ J, Liu $X, X i a ~ X, L i ~ N, B i X$. Shikonin induces apoptosis in the human gastric cancer cells HGC-27 through mitochondria-mediated pathway. Pharmacognosy Magazine,2015;11(42):250

25. Tan W, Lu J, Huang M, Li Y, Chen M, Wu G, et al. Anti-cancer natural products isolated from chinese medicinal herbs. Chin Med. 2011; 6: 27.

26. Wang $\mathrm{P}$, Lan $\mathrm{C}$, Xiong $\mathrm{S}$, Zhao X, Shan $\mathrm{Y}, \mathrm{Hu}$ R, et al. HIF1alpha regulates single differentiated glioma cell dedifferentiation to stem-like cell phenotypes with high tumorigenic potential under hypoxia. . Oncotarget 2017; 8(17): 28074-92.

27. Robertson ED, Semenchenko K Fau - Wasylyk B, Wasylyk B. Crosstalk between Mdm2, p53 and HIF1-alpha: distinct responses to oxygen stress and implications for tumour hypoxia. Subcell Biochem. 2014; 85: 199-214.

28. Azoitei N, Becher A, Steinestel K, Rouhi A, Diepold K, Genze F, et al. PKM2 promotes tumor angiogenesis by regulating HIF-1alpha through NF-kappaB activation. Molecular cancer therapeutics. 2016;15(1):3

29. Xu Q, Liu LZ, Yin Y, He J, Li Q, Qian X, et al. Regulatory circuit of PKM2/NF-[kappa]B/miR-148a/152-modulated tumor angiogenesis and cancer progression. Oncogene. 2015; 34: 5482-93.

30. Li X, Fan XX, Jiang ZB, Loo WT, Yao XJ, Leung EL, et al. Shikonin inhibits gefitinib-resistant non-small cell lung cancer by inhibiting TrxR and activating the EGFR proteasomal degradation pathway. Pharmacol Res. 2017; 115: 45-55.

31. Casimiro M, Sante GD, Loro E, Pestell T, Bisetto S, Velasco-Velázquez M, et al. Abstract B10: Cyclin D1 restrains oncogene-induced autophagy via phosphorylation of LKB1. Cancer Research. 2016; 76: B10-B10.

32. Yan G-J, Yu F, Wang B, Zhou H-J, Ge Q-Y, Su J, et al. MicroRNA miR-302 inhibits the tumorigenicity of endometrial cancer cells by suppression of Cyclin D1 and CDK1. Cancer Letters. 2014; 345: 39-47.
33. Chen J, Bai M, Ning C, Xie B, Zhang J, Liao H, et al. Gankyrin facilitates follicle-stimulating hormone-driven ovarian cancer cell proliferation through the PI3K/AKT/HIF-1[alpha]/cyclin D1 pathway. Oncogene. 2016; 35: 2506-17.

34. Yeo D, Phillips P, Baldwin GS, He H, Nikfarjam M. Inhibition of group 1 p21-activated kinases suppresses pancreatic stellate cell activation and increases survival of mice with pancreatic cancer. International journal of cancer. 2017; 140: 2101-11.

35. Prigione A, Rohwer N, Hoffmann S, Mlody B, Drews K, Bukowiecki R, et al. HIF1alpha modulates cell fate reprogramming through early glycolytic shift and upregulation of PDK1-3 and PKM2. Stem cells (Dayton, Ohio). 2014; 32: 364-76. 